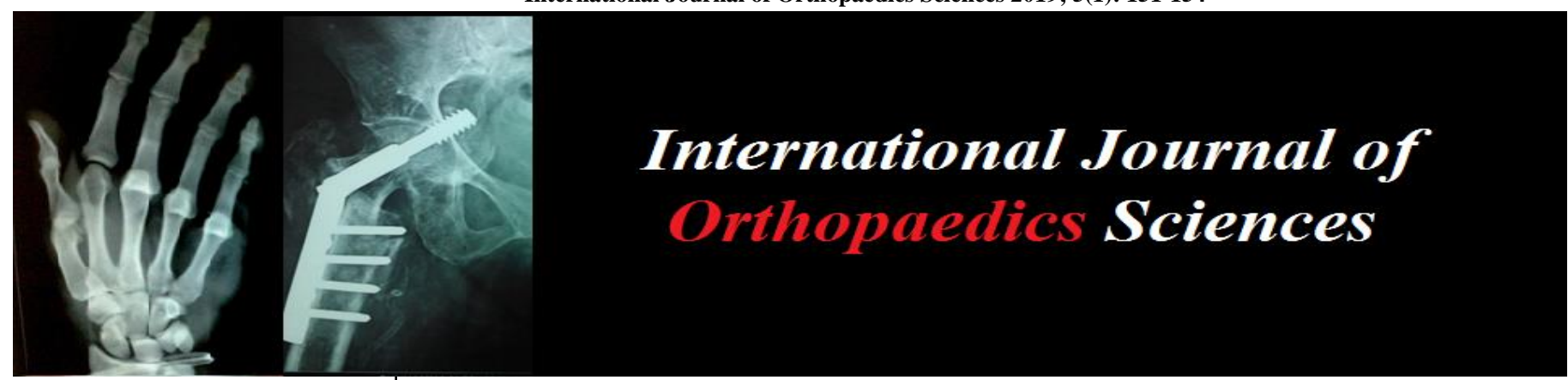

ISSN: $2395-1958$

IJOS 2019; 5(1): 151-154

(C) 2019 IJOS

www.orthopaper.com

Received: 19-11-2018

Accepted: 23-12-2018

\section{Mohd Nazim}

Assistant Professor, Department of orthopaedics, Gulbarga

Institute of Medical Sciences,

Kalaburgi, Karnataka, India

Dr. Vijay Kumar Rathod

Assistant professor, department

of Ortho, GIMS, Kalaburgi,

Karnataka, India

\section{Ulnar lengthening osteotomy in the treatment of Kienbock disease: A prospective case series}

\author{
Mohd Nazim and Dr. Vijay Kumar Rathod
}

DOI: https://doi.org/10.22271/ortho.2019.v5.i1c.28

\section{Abstract}

Introduction: Kienbock disease or avascular necrosis of the carpal bone, lunate, is not an uncommon disease but its treatment is still controversial. Treatment ranges from conservative management to proximal row capectomy and arthrodesis. Most commonly used procedure is radial shortening. This is a prospective study of Kienbock disease in which patients were managed by ulnar lengthening. Both the procedures unloads the lunate fossa and redistributes load to the scaphoid, and are indicated in early disease with negative ulnar variance.

The purpose of this study is to evaluate the functional outcome and disease progression; and to compare it with the available literature and other modalities of treatment.

Materials and Methods: 8 patients were operated for Kienbock disease by ulnar lengthening, between January 2008 to June 2012 in our institution. Patients who didn't improve after a conservative treatment and stage 2 to stage $3 \mathrm{~b}$ were included in the study. Patients with stage 4 , with other systemic diseases are excluded from the study.

All the patients were evaluated pre-operatively clinically and radiologically and staged according to Litchman et al. Patients were followed up with a mean of 30 months post operatively for clinical and functional outcome. Assessment was done by quick DASH score, Visual analogue scale, and radiologically, for progression of the disease in subsequent follow up.

Results: Of 8 patients, 7 were followed-up for a mean of 30 months, sof which 6 patients were having good clinical (60\% improvement in Quick DASH score) and radiological improvement, and 1 complained of no improvement subjectively, with no complication of non-union.

Summary: Ulnar lengthening osteotomy is a less demanding yet a good option in mild and moderate cases of Kienbock disease, who does not respond to conservative management. Our study did not experience complications like non-union as per previous literatures, and the functional outcome was good.

Keywords: kienbock disease, osteotomy, litchmen et al

\section{Introduction}

Kienböck disease is a painful disorder of the wrist of unknown cause in which radiographs eventually show osteonecrosis of the carpal lunate. It occurs more frequently between the ages of 15 and 40 years and in the dominant wrist of men engaged in manual labor ${ }^{[1]}$. Armistead et al., using CT, showed in some patients occult fractures of the lunate. Untreated, the disease usually results in fragmentation of the lunate, collapse with shortening of the carpus ${ }^{[1]}$. Because the natural course of Kienböck disease is unpredictable, the treatment of established Kienböck disease cannot be rigidly prescribed. Treatment ranging from conservative likes plaster cast immobilisation, to proximal row carpectomy and wrist arthrodesis.

The operative treatment options: 1) For Early disease radial shortening, ulnar lengthening, capitate-hamate fusion, Scaphod Trapezium Trapezoid (STT) fusion, and vascular bundle implantation. 2) For late disease proximal row carpectomy and wrist arthrodesis ${ }^{[2]}$.

Most commonly used procedure is radial shortening. But lots of controversy regarding its management persists. No ideal treatment till now which provides an optimum results and hold an upper hand over other treatment options. According to previous studies both radial shortening and ulnar lengthening results in similar functional outcome in long term follow up, but complications like non-union is more with ulnar lengthening. Both the procedures unload the lunate fossa and redistributes load to the scaphoid, and are indicated in early disease
Correspondence Mohd Nazim

Assistant Professor, Department of orthopaedics, Gulbarga Institute of Medical Sciences, Kalaburgi, Karnataka, India 
With negative ulnar variance. Ulnar variance is measured by drawing transverse line at the level of the lunate fossa, and a second transverse line at the level of the ulnar head, the difference indicates ulnar variance (Normal Value $0.9 \mathrm{~mm}$. ranging from 2.3 to $4.2 \mathrm{~mm}$.). Patients with Kienböck's disease $78 \%$ are found have ulnar minus variance, compared with $23 \%$ in the general population ${ }^{[4]}$.

Our study deals with kienbock disease patients managed by ulnar lengthening osteotomy. The purpose of this study is to evaluate the functional outcome and disease progression; and to compare it with the available literature and other modalities of treatment.

\section{Materials and Methods}

8 patients were operated for Kienbock disease by ulnar lengthening, between January 2008 to June 2012 in our institution. Patients who didn't improve after a conservative treatment and stage 2 to stage $3 \mathrm{~b}$ were included in the study. Patients with stage 4, with other systemic diseases are excluded from the study.

All the patients were evaluated pre-operatively clinically and radiologically and staged according to Litchman et al. Patients were followed up with a mean of 30 months post operatively for clinical and functional outcome. Assessment was done by quick DASH score, Visual analogue scale, and radiologically, for progression of the disease in subsequent follow up. Radiograph includes standard AP and lateral views as well as oblique view. In our study 2 patients were in stage 2 and 6 were in stage $3 b$.

\section{Litchman et al staging of Kienbock disease}

\begin{tabular}{|c|c|}
\hline stages & Radiological Features \\
\hline 1 & $\begin{array}{c}\text { normal except for the possibility of either a linear or a } \\
\text { compression fracture }\end{array}$ \\
\hline 2 & definite density changes apparent in the lunate \\
\hline 3A & collapse of entire lunate without fixed scaphoid rotation \\
\hline 3B & collapse of entire lunate with fixed scaphoid rotation \\
\hline 4 & $\begin{array}{c}\text { stage III with generalized degenerative changes in the } \\
\text { carpus }\end{array}$ \\
\hline
\end{tabular}

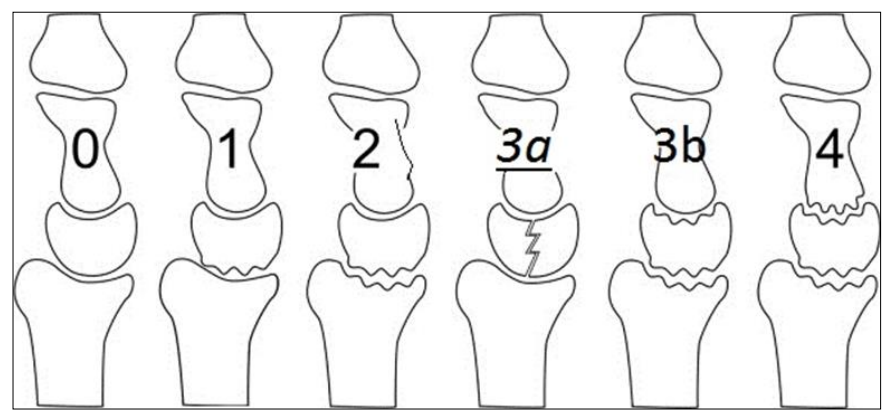

Litchman et al staging (diagrammatic representation)

\section{Operative Procedure}

Patients with severe symptoms and in who did not respond to conservative management were taken up for surgery after complete clinical evaluation and radiological staging.

Patient in supine or lateral position, general anaesthesia or regional block (brachial) used for the procedure. The ulna was approached with a dorsal incision, after soft tissue dissection, ulnar osteotomy preformed at the junction of proximal threefourth and distal one-fourth, after the placement of the LCDCP. The amount of lengthening is determined preoperatively by negative ulnar variance value plus $1 \mathrm{~mm}$. Fixed with 6 holed LC-DCP and cortical screws not in compression mode. Bone graft is harvested from the Olecranon process of the same side.

Post-operatively immobilised in a long arm cast for 3 weeks, followed by active range of movements of elbow and wrist.

\section{Follow-Up}

Follow-up was done 1 month, 3 month, 6month, $1^{\text {st }}$ and $2^{\text {nd }}$ year post operatively by clinical and radiological assessment for

1. Stage of the disease and its progression,

2. Functional improvement by Quick DASH score and Visual analog scale

3. Complications like infection, non-union, graft site morbidity

4. Regain of normal activity

5. $\quad$.....

And the results were categorised as excellent, good and poor out come on the basis of VAS scale and Quick DASH scores.

\section{Results}

Of 8 patients, 2 patient lost follow-up, remaining 6 were included in the study, and followed up for a mean period of 30 months.

\section{Demographic data}

\begin{tabular}{|c|c|}
\hline Variables & No. Or mean (\%) \\
\hline Total patients followed up & 6 \\
\hline Male: female Ratio & $2: 1$ \\
\hline Dominant hand & $5(83 \%)$ \\
\hline Bilateral involvement & 0 \\
\hline Mean age & 28years \\
\hline
\end{tabular}

Clinical evaluation. At the last follow-up 6 patients were asymptomatic (VAS grade 0) and 1 reported mild pain (with VAS grade 2). The mean\ Quick DASH score was 14.94 points (0 to 35). The mean ROM improved from $76.8^{\circ}\left(30^{\circ}\right.$ to $107^{\circ}$ ) preoperatively to $100.5^{\circ}\left(65^{\circ}\right.$ to $\left.140^{\circ}\right)$, at the latest follow-up; this represents a significant mean increase of $23.7^{\circ}$ Compared with the unaffected side.

Radiological evaluation. The objective of levelling the distal radio ulnar joint (DRUJ) was achieved. The ulnar variance increased from a mean negative value of $0.63 \mathrm{~mm}(-1.23$ to $0.00)$ pre-operatively to a mean positive value of $1.11 \mathrm{~mm}$ (0.53 to 1.62). At the three-month follow-up evaluation all osteotomies showed radiological signs of union. There was no significant difference between carpal height ratios both preoperatively and at follow-up. No wrist had radiological evidence of carpal degeneration

Stages II and IIIA versus stage IIIB. Patients in stages II and IIIA were compared with patients in stage IIIB. Clinically, patients in both groups had an increase in ROM, but no significant differences were found between groups in the other parameters and Radiological

Complications. No major complications were reported at the last follow-up. There were no non-union and no infections. No patient reported ulnar-sided wrist pain.

\section{Pitfalls}

The study group very small to give any conclusive results or 
recommendation for management.

There was no control group, but the results were compare with the literature of same procedure and other procedure like radial shorting. 2 patient (25\%) lost follow up

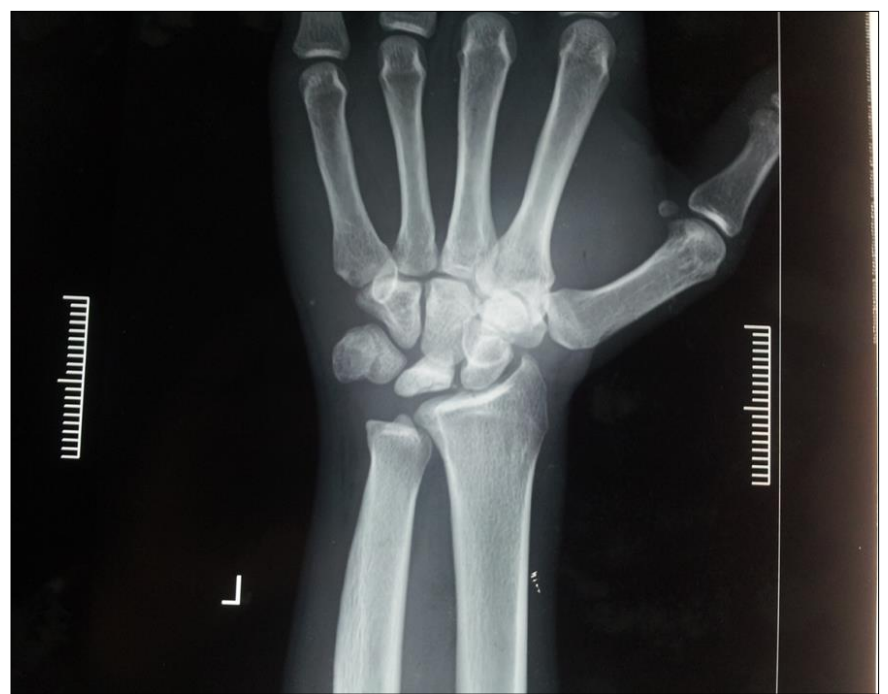

X ray of left wrist AP view Kienbock disease, litman's stage 3a (patient 1)

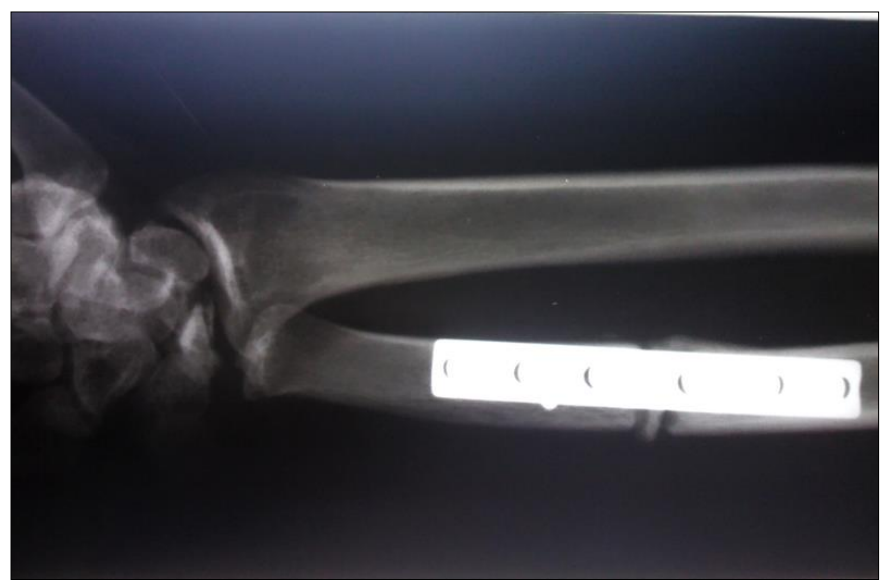

X-ray AP veiw of distal forearm with wrist taken postoperatively showing ulnar osteotomy site, with bone graft in place, with 6 hole small LC-DCP fixation of osteotomy (patient 1)

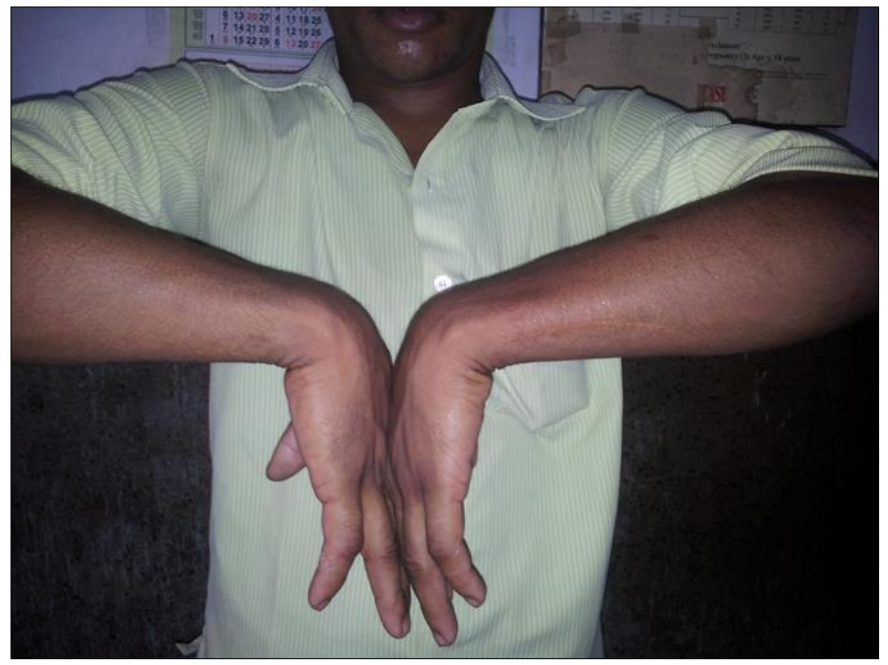

Range of motion of wrist joint during follow-up period, 6month postoperatively (patient 1 )
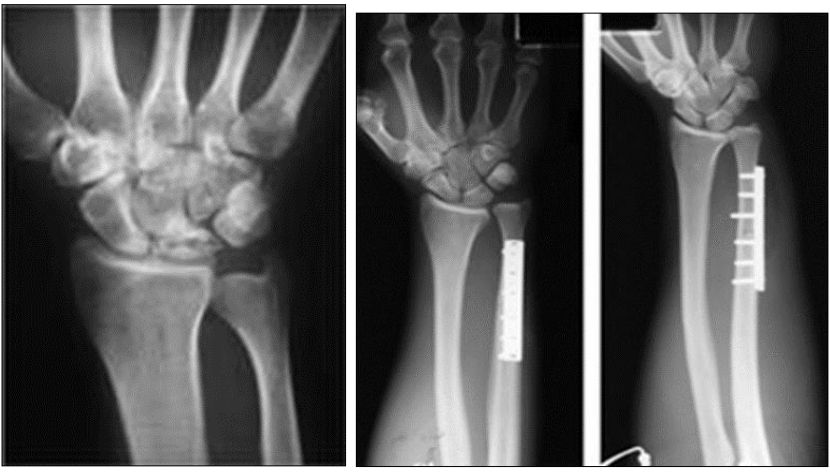

Patient 2 preoperative $\mathrm{x}$ ray and post-operative $\mathrm{x}$ ray

\section{Discussion}

According to previous studies Both redial shortening and ulnar lengthening results in similar functional outcome in long term follow up, but complications like non-union is more with ulnar lengthening. Previous studies reported that radiological improvement is minimal or there is no further increase in stage of the disease following treatment, however there is no correlation between post-operative clinical and radiological improvement.

Persson in 1950 reported a series of patients in whom he lengthened the ulna for Kienböck disease. Axelsson and Moberg observed these patients for several years. They found 16 patients who had been operated on some 20 years previously, and all but one had been able to continue with manual labor after the operation. Even in one who had pain, the disease process seemed to have been halted. Subsequently, Armistead et al. performed the ulnar lengthening operation for Kienböck disease, reporting 20 cases in 1982. Three nonunions required a second plating and bone grafting; 18 of the 20 patients had pain relief ${ }^{[1]}$.

In our study 6 patients managed by ulnar lengthening are followed up for a mean period of 30 months postoperatively. Of which 5 patients $(83 \%)$ were having good clinical $(60 \%$ improvement in Quick DASH score) and radiological improvement, and 1(33\%) complained of no improvement subjectively. There was no significant progressive lunate collapse in our patients. However, carpal height ratio didn't improve significantly.

All the patients achieved radiological union at mean of 6 months, with no case of non-union in this series. This is mainly due to the accurate and stable fixation of the osteotomy site achieved per operatively, as well as no complication related to the graft donor site. 1 patient complaining of mild residual pain at the donor site.

\section{Summary}

Ulnar lengthening osteotomy is a less demanding yet a good option in mild and moderate cases of Kienbock disease, who does not respond to conservative management. Our study did not experience complications like non-union as per previous literatures, and the functional outcome was good

However, the surgeon cannot ignore the fact that patients in later stages may have a worse clinical outcome, especially less grip strength, and more disability, and this should be explained when choosing this procedure for patients with advanced stages of the disease.

\section{References}

1. Campbell's operative orthopaedics, $11^{\text {th }}$ edition, 2007, 5657.

2. Wheeless' textbook of orthopaedics. 
3. Tadayoshi Watanabe, MD Masatoshi Takahara, MD $\mathrm{PhD}$, Hiroyuki Tsuchida MD, PhD, Shinichi Yamahara, MD, Noriaki Kikuchi, MD, PhD, Toshihiko Ogino, MD, $\mathrm{PhD}$ Long-Term Follow-up of Radial Shortening Osteotomy for Kienböck Disease J Bone Joint Surg Am, 2008; 90(8):1705-1711. DOI: 10.2106/JBJS.G.00421.

4. J Bone Joint Surg Br February. Portugal Clinical and radiological results following radial osteotomy in patients with Kienböck's disease, 2012.

5. Ray B, Brmistead MD, Ronald L, Linscheid MD, James H, Dobyns MD, Robert D. beckenbaugh, M.D, Rochester, Minnesota From the Department of Orthopedics, Mayo Clinic and Mayo Foundation, Rochester Ulnar Lengthening in the Treatment of Kienbock's Disease, 1982 by The Journal of Bone and Joint Surgery, 2012.

6. Wasaki NL, Minami A, Oizumi N, Suenaga N, Kato H, Minami M. Radial osteotomy for late-stage Kienbock's disease: Wedge osteotomy versus radial shorteningJ Bone Joint Surg [Br]. 2002; 84-B:673-677.

7. Salmon J, Stanley JK, Trai IA. IKienbock's disease: conservative management versus RADIAL shortening, J Bone Joint Surg [Br]. 2000; 82-B:820-823

8. Rodrigues-Pinto R, Freitas D, Costa LD, Sousa R, Trigueiros M, Lemos $\mathrm{R}$, et al. A review article, J Bone Joint Surg [Br]. 2012; 94-B:222-226.

9. Schuind F, Eslami S, Ledoux P, Kienbock's disease ...lunate. Lichtman classified Kienbock disease into five stages J Bone Joint Surg [Br]. 2008; 90-B:133-139.

10. Iwasaki N, Minami A, Oizumi N, Suenaga N, Kato H, Minami M. Radial osteotomy for late-stage Kienbock's disease: Wedge osteotomy versus radial shortening, J Bone Joint Surg [Br]. 2002; 84-B:673-677.

11. Alford Dornan. The results of treatment in kienbock's disease, J Bone Joint Surg [Br]. 1949; 31-B:518-520. 